\title{
A novel mixed matrix membrane of phenolphthalein hydrazide and polysulfone for the detection of copper ions in environmental water samples.
}

SREERAMAREDDYGARI, M., KEMPAHANUMAKKAGARI, S., MAHESH, P., THIPPESWAMY, R., DEVARAMANI, S., MARDI, R.R., CARLOS, F. and GOVINDAPPA, $B$. 


\section{A Novel Mixed Matrix Membrane of Phenolphthalein Hydrazide and Polysulfone for the Detection of Copper Ions in Environmental Water Samples}

Muralikrishna Sreeramareddygari,a,b Sureshkumar Kempahanumakkagari,c Padaki

Mahesh,b

Ramakrishnappa Thippeswamy, d Samrat Devaramani,e Radhakrishna Reddy Mardi,f

Fernandez Carlos,g

and Banuprakash Govindappan

aPilot Plant Development and Training Institute, King Mongkut's University of

Technology Thonburi, Bangkhuntien-chaitalay Road,

Thakam, Bangkok 10150, Thailand

bCenter for Nano and Material Sciences, Global Campus, Jain University, Jakkasandra

$(\mathrm{P})$, Kankapura $(\mathrm{T})$, Bengaluru 560001,

Karnataka, India

¿School of Basic and Applied Sciences, Department of Biological Sciences, Dayananda

Sagar University, Shavige Malleshwara Hills,

Kumaraswamy Layout, Bengaluru 590078, Karnataka, India

aDepartment of Chemistry, BMS Institute of Technology and Management, Avalahalli, Doddaballapura Main road, Yelahanka,

Bengaluru 560064, Karnataka, India; swadheshi26@gmail.com (for correspondence)

eDepartment of Chemistry, Dayananda Sagar College of Engineering, Shavige

Malleshwara Hills, Kumaraswamy Layout, Bengaluru

590078, Karnataka, India

fDayananda Sagar Academy of Technology and Management, Udayapura, Kanakapura

Road, Opp. Art of Living, Bengaluru 560082,

Karnataka, India

gSchool of Pharmacy and Life Sciences, Robert Gordon University, Garthdee Road,

AB10 7GJ, Aberdeen, United Kingdom

hDepartment of Chemistry, SJB Institute of Technology, Dr.Vishnuvardan road, Kengeri, Bengaluru 560060, Karnataka, India

The novel membrane test strip of phenolphthalein hydrazide( $\mathrm{PH})$-polysulfone has been designed and demonstrated for $\mathrm{Cu} 2+$ ions detection. Before finding performance of membrane the studies with $\mathrm{PH}$ alone has been performed. Aqueous solutions of $\mathrm{PH}$ are colorless, but upon interaction with $\mathrm{Cu} 2+$ ions become pink (when $8.2 \leq \mathrm{pH} \geq 12$ ). The colorimetric change is initiated by the coordination of $\mathrm{Cu} 2+$ with $\mathrm{PH}$ as a polydentate ligand, opening the spirolactam ring that subsequently hydrolyses releasing phenolphthalein (P). Further membrane was 
preloaded with $\mathrm{PH}$ that could be used as a simple, low cost, and portable sensor for $\mathrm{Cu} 2+$ ions in environmental water samples. Given the detection limits of this sensor, a maximum response would warn the tester that $\mathrm{Cu} 2+$ concentrations were above safe drinking regulation values. Approximate concentrations can be determined via a color comparison chart. Different metal ions were tested in order to determine the chemo dosimeters specificity, of the 12 tested ions only $\mathrm{Hg} 2+$ induced a similar molecular transformation (i.e., $\mathrm{PH}$ to $\mathrm{P}$ ). The chemo dosimeter allows the quantification of $\mathrm{Cu} 2+$ ions in the linear dynamic range of $0-22 \mu \mathrm{M}$. The Sandell sensitivity, limit of detection, limit of quantification, and relative standard deviation were found to be $0.276 \mu \mathrm{g} / \mathrm{mL} / \mathrm{cm} 2,0.279 \mu \mathrm{M}, 1.674 \mu \mathrm{M}$, and $0.682 \%$ respectively.

Keywords: chemo dosimeter, phenolphthalein hydrazide, polysulfone membrane, naked eye sensor, phenolphthalein

\section{INTRODUCTION}

The polymeric membranes have their own intense in the field. Membrane technology is emerging in the field of separation and water purification [1]. The application of the polymericmembrane is not restricted to water purification [2]. It has its own identity in versatile applications such as fuel cell[3], super capacitor [4], packing material [5], drug delivery [6],biological application [7], and absorption as well as adsorption[8]. However, applications of membrane are increasing in many other fields. The property performance and application of the membrane can be tuneable by the compositing of the polymers [9].In recent years the design, synthesis and use of chemosensory a highly active research area due to extensive applications in medical, environmental, and biological sectors [10].For example, the detection and quantification of heavy metals gain extreme importance due to the fine balance between the necessity in many biological functions and harmful effects when in excess [11]. Among the various heavy metal ions, copper plays a significant role in many physiological processes. Though it is found to be an essential trace element, ranked third in abundance in the human body behind iron and zinc [12]. Recent years, the increase in copper concentrations within water bodies due to the discharge of industrial effluents into the environment [13]. The US Environmental Protection Agency has set the safety limit of copper in drinking water at $1.3 \mathrm{ppm}$ [14]. Several instrumental techniques are currently routinely used for the detection and quantification of copper ions in samples, these include atomic absorption spectrometry(AAS), inductively coupled plasma atomic emission spectrometry and inductively coupled plasma mass spectrometry. However, their use is limited owing to the requirement of expensive capital costs (e.g., equipment and laboratory space), the need for specialist training and time consuming procedures[15]. Alternatives such as spectroscopic techniques have become increasing popular for sensing copper owing to theirability to detect species fast and effectively, at low cost and in some cases via the naked eye. Consequently, the development of colorimetric chemosensors based on perceptible color changes in the presence of copper ions has emerged as an active area of research [16-18].Many of these reported sensors are structurally complicated, requiring tedious synthetic procedures and can require an organic co-solvent due to their low solubility in aqueous media. Therefore, the development of a simple, easily synthesisable, and watersoluble chemosensor is highly desirable. Recently, xanthene derivatives have emerged as promising materials for sensing various analytes (as both absorbers and emitters), due to their binding and optical properties [19]. Rhodamine B hydrazidewas the first example of a fluorescent probe for the detection of $\mathrm{Cu} 2+$ ions reported [20]. Since then, numerous other molecular probes for different cations and anions, including bioanalytes, have been reported 
[20]. Within the cited literature, absorption or emission changes of the sensing molecule, induced by the analyte, proceed either via reversible coordination(e.g., ion displacement) or an irreversible structural transformation(e.g., cation induced ring opening). Following the latter approach, the xanthene moiety PH was designed, synthesized and tested for the colorimetric sensing of divalent copper ions in aqueous media. To our knowledge, phenolphthalein has not been used as metal ion sensor in this way. However, there are some reports in the literature, where by cyclodextrin and similar derivatives have been used as $\mathrm{pH}$ sensors [21]. The chemodosimeter proposed in this article has the advantage over others previously synthesized (such as Rhodamine B hydrazide, which can detect concentrations to at least one order of magnitude greater) [19]. Additionally, because PH undergoes a visible adsorption change, the probe is introduced in the polymeric membrane to use as detection strip. The data presented within this article illustrates the first useof the novel molecular probe $\mathrm{PH}$, as a chemodosimeter forCu2+ ions at micro-molar concentrations in aqueous samples. Furthermore, the probe has been embedded into a polymermembrane and used as a naked eye solid-state sensor strip, allowing the fast and effective measurement of $\mathrm{Cu} 2+$ ions in various environmental water samples at the source.

\section{EXPERIMENTAL}

Instrumentation

All absorbance measurements were made with a Shimadzu Spectrophotometer (model: UV1800, using a Tungsten lamp)using $1 \mathrm{~cm}$ path-length quartz cuvettes. All fluorescence studies were done using Shimadzu Spectroluorimeter (modelRF5301PC). Proton nuclear magnetic resonance (1H NMR)measurements were performed using a Bruker $400 \mathrm{MHz}$ spectrometer with chemical shifts measured against a tetramethylsilane internal standard. Samples were analyzed in $\mathrm{CDCl} 3(99.8 \%$ D). Mass spectrometry was performed using a Thermo Finnigin Deca QXP Mass Spectrometer. A Shimadzu Atomic Absorption Spectrophotometer (ASS, model: AA-7000) was used for validating environmental water sample's $\mathrm{Cu} 2+$ concentrations, where by their concentration was determined from calibration plot constructed using copper standard solution's purchased from the Merck LTD, India. All solutions were aspirated into the flame and the absorbance recorded. $\mathrm{pH}$ measurements were carried out using a Systronics digital $\mathrm{pH}$ meter(model 802) that was calibrated using commercial standard solutions of $\mathrm{pH} 7$ and 9. Marvin Sketch was used for drawing, displaying and characterizing chemical structures, substructures, and reactions, Marvin 5.10.2, 2012, Chem Axon (http://www.chemaxon.com).

\section{Chemicals and Reagents}

Phenolphthalein and polysulfone (35,000 Da) were purchased from Sigma-Aldrich. Hydrazine hydrate, magnesium sulphate ( $>99 \%)$, sodium tetraborate $(>98.0 \%)$, and ethanol(HPLC grade) were purchased from SD Fine Chem. Ltd.N-methyl pyrrolidine (reagent grade), copper(II) chloride AR grade, acetonitrile (99\%), acetic acid (99\%), ortho-phosphoricacid (88\%), and sodium hydroxide (97\%) were all purchased from Merck, Mumbai, India. All reagents were used without further purification. Water was doubly distilled in-house. PH solutions $(10 \mathrm{mM})$ were prepared by dissolution in 1:10 acetonitrile/water (vol/vol \%). Rhodamine B phenyl hydrazide $(10 \mathrm{mM})$ was prepared by dissolving $0.532 \mathrm{~g}$ in $1: 10$ acetonitrile/water ( $\mathrm{vol} / \mathrm{vol} \%)$ [22]. Standard $\mathrm{Cu} 2+$ solutions were prepared by dissolving $\mathrm{CuCl} 2$ (various concentrations) in distilled water. Britton-Robinson buffer solutions of $\mathrm{pH}$ 6-12 were prepared by using solutions of acetic acid $(20 \mathrm{mM})$, orthophosphoric acid $(20 \mathrm{mM})$, sodium tetraborate $(20 \mathrm{mM})$, and 
sodium hydroxide (400 mM). Sample Collection and Preparation Environmental water samples were collected from various lakes of different localities in polyethylene bottles. These samples were filtered to remove suspended particles followed by treatment with activated charcoal in order to decolorize, before performing spectroscopic analysis.

\section{ANALYTICAL PROCEDURES FOR Cu2+ IONS DETERMINATION}

\section{Colorimetric Procedure (Proposed Method)}

Aliquots of standard solutions of $\mathrm{Cu} 2+$ ions (so that the final concentration should be in the range of $0-22 \mu \mathrm{M}$ ) were taken in $10 \mathrm{~mL}$ standard volumetric flasks. To this, $250 \mu \mathrm{L}$ of $\mathrm{PH}$ solution(10 mM) was added, along with $1 \mathrm{~mL}$ of Britton-Robinson buffer solution, $\mathrm{pH} 12$. Then the volumetric flasks were allowed to stand for $5 \mathrm{~min}$ for the development of color, finally made up to the mark $(10 \mathrm{~mL})$ using distilled water and then absorbance was measured at 478 $\mathrm{nm}$. Using the absorbance values of the standard solutions the standard curve was drawn and limit of detection was calculated. Then the known aliquots of real sample solution after pre treatment as discussed in earlier section was taken and same procedure was fallowed as in the case of standard solutions and absorbance values obtained were used to determine the $\mathrm{Cu} 2+$ ions of the solutions.

\section{Fluorimetric Procedure}

The $\mathrm{Cu} 2+$ ions in the natural samples have been determined by the fluorimetric method in order to compare the results of the proposed method. All the water samples were diluted200 folds before analyzing by this method as their concentrations were very high. The aliquots of standard $\mathrm{Cu} 2+$ ion solution $(0.01-0.1 \mu \mathrm{M})$ were taken in $10 \mathrm{~mL}$ standard flask, to this $1 \mathrm{~mL}$ of $\mathrm{RBPH}$, and $1 \mathrm{~mL}$ of Robinson buffer solution of $\mathrm{pH} 6$ were added and kept aside for $2 \mathrm{~min}$ at room temperature. Then the flasks were made up to the mark with distilled water and the developed fluorescent intensity of the solutions were measured $(\lambda \mathrm{ex} / \mathrm{em}=561 / 580 \mathrm{~nm})$ [22].The linear graph were drawn with fluorescent intensity values against concentration of $\mathrm{Cu} 2+$ ions and concentrations of the different water samples were calculated. The analysis results of this procedure are given in table 1 and comparison of the results with proposed method were discussed in application section.

\section{AAS Procedure}

The $\mathrm{Cu} 2+$ ions in the natural samples have been determined by the AAS technique in order to compare the results of the proposed method. Aliquots of standard $\mathrm{Cu} 2+$ solutions (concentration range $0-22 \mu \mathrm{g}$ ) were transferred into $10 \mathrm{~mL}$ calibrated standard flasks and made up to the mark with distilled water and analyzed by the AAS method for the construction of calibration plot. The analysis results of above procedures were given in table 2 and comparison of the results were discussed in application section.

\section{SYNTHESIS}

\section{Synthesis of $\mathrm{PH}$}

PH was synthesized in a standard round-bottomed flask $(250 \mathrm{~mL})$ by dissolving $\mathrm{P}(1 \mathrm{~g}, 3 \mathrm{mM})$ in ethanol $(20 \mathrm{~mL})$. To this solution, excess hydrazine hydrate $(2 \mathrm{~mL})$ was added drop-wise and the reaction refluxed for $6 \mathrm{~h}$. Subsequently, the solvent was then removed via evaporation and the residue dissolved in ethyl acetate $(50 \mathrm{~mL})$. The solution was then washed with saturated 
aqueous bicarbonate solution three times. The ethyl acetate layer was collected and treated with anhydrous magnesium sulfate to remove any trace water. The solution was filtered and the solvent removed via evaporation. The final product was recrystallized using methanol, and characterized by electrospray-ionization mass-spectrometry (ESI-MS)and 1H NMR. ESI-MS calculated mass $[\mathrm{M}+\mathrm{H}]+=333.3$, obtained mass $[\mathrm{M}+\mathrm{H}]+=333.2$ (Fig. SD1, Supplementary

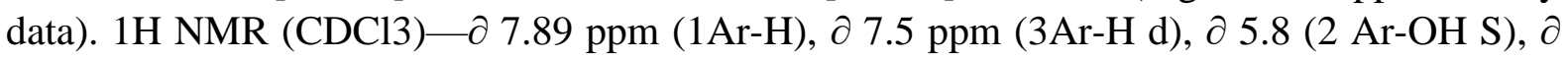
9.5 ppm (2Ar-HS), $\partial 6.7$ ppm (4Ar-Hd), $\partial 6.9$ ppm (4Ar-Hd) (Fig. SD8, Supplementary data). The synthetic pathway is summarized in Scheme 1.Synthesis of the Polymer Membrane Strip for Naked Eye Detection of $\mathrm{Cu} 2+$ Ions $\mathrm{PH}(0.5 \mathrm{~g})$ was dissolved in N-methyl pyrrolidine (5 $\mathrm{mL})$ before adding polysulfone $(0.5 \mathrm{~g})$ to produce a viscous solution, which was stirred for about $24 \mathrm{~h}$ at 60_C. The resulting solution was then degassed (N2) and allowed to stand for 1 $\mathrm{h}$ before being filtered, using G4 sand filters. The viscous liquid was then poured onto a glass plate and spread with a glass rod to a thickness of $0.20 \mathrm{~mm}$. The layer was left to dry at room temperature for $40 \mathrm{~h}$, before separating the membrane from the glass plate and cutting into uniform sized strips $(2 \times 0.8 \mathrm{~cm})$.

\section{RESULTS AND DISCUSSION}

\section{Spectral Properties of $\mathrm{P}$ and $\mathrm{PH}$}

The spectral properties of $\mathrm{P}$ vary greatly with $\mathrm{pH}$ due to resulting molecular rearrangements that alter the extent of conjugation within the molecule. The structures of $\mathrm{P}$ at various $\mathrm{Ph}$ conditions are shown in Scheme 2. $\mathrm{P}$ is colorless between $\mathrm{pH}-1$ and 8.2 due to its spiro-closed structure. In the $\mathrm{Ph}$ range 8.2-12, $\mathrm{P}$ takes on a spiro-open structure and exhibits a pink color resulting from the extended conjugation between the phenyl rings. Above $\mathrm{pH} 12$, phenolphthalein becomes colorless due to carbinol formation and the associated loss of extended conjugation. The spectral properties of $\mathrm{PH}$ were studied in an acetonitrile: water system (1:10), because of the low solubility in pure water. The resulting solution is colorless within the $\mathrm{pH}$ range 1-12 (Britton-Robinson buffer solutions) and possesses minimal absorption due to its spiro-closed structure(as for $\mathrm{P}$ ). Gradual addition of $\mathrm{Cu} 2+$ ions to the solution causes the emergence of a pink color when within the $\mathrm{Ph}$ range 8.2-12. The absorbance values increase linearly at $478 \mathrm{~nm}$ between 0 and $22 \mu \mathrm{M} \mathrm{Cu} 2+$ concentrations (Figure 1).The observed color is due to the release of $\mathrm{P}$ upon interaction with $\mathrm{Cu} 2+$, which adopts the spiroopen structure within this $\mathrm{pH}$ range. Various other metal ions were tested, with only one other inducing a color change within the same experimental conditions, $\mathrm{Hg} 2+$. This induced a color change at $\sim 20 \%$ lower intensity than that of the $\mathrm{Cu} 2+$ ions (Figure 2). The $\mathrm{Hg} 2+$ mechanism can be explained by the interactions of elements within the "copper triangle" [23].

Sensing Mechanism

It is well known that many xanthene-based molecules are colorless and non fluorescent in their spiro-closed form, but colored and highly fluorescent in the spiro-open form [24].Similarly, $\mathrm{PH}$ is colorless due to its spiro-closed structure but after interaction with $\mathrm{Cu} 2+$ ions release the pink colored spiro open form of $\mathrm{P}$, when between $\mathrm{pH} 8.2-12$. The coordination of $\mathrm{Cu} 2+$ ions with the hydrazide group results in the formation of $\mathrm{Cu} 2+$ polydentate complex, this then undergoes reductive hydrolysis to release the P. The final step was confirmed by the ESI-MS studies of the probe and the probe in the presence of $\mathrm{Cu} 2+$ in Britton-Robinson buffer solution ( $\mathrm{pH}$ 12). $\mathrm{PH}$ has a protonated molecular mass of $333.2 \mathrm{Da}$ (Figure SD1, Supplementary data), whereas reaction with $\mathrm{Cu} 2+$ produces protonated molecular mass of $321.3 \mathrm{Da}$ (Figure SD2, 
Supplementary data) corresponding to the protonated molecular mass of P. Based on the above studies, a probable reaction mechanism for the conversion of $\mathrm{PH}$ to $\mathrm{P}$, via the addition of $\mathrm{Cu} 2+$ is given in Scheme 3. Cu2+ Sensing Optimization Parameters that affect the interaction between $\mathrm{PH}$ and $\mathrm{Cu} 2+$ ions have been studied in order to optimize the sensing capabilities of phenolphthalein hydrazide.

\section{Concentration}

The effect of PH concentration on absorbance was studied whilst keeping all other parameters constant. In a set of volumetric flasks $(10 \mathrm{~mL})$, Britton-Robinson buffer solution $\mathrm{pH} 12(1 \mathrm{~mL})$ and $\mathrm{CuCl} 2(25 \mu \mathrm{M})$ were prepared. To this, different concentrations of $\mathrm{PH}(0.1-0.7 \mathrm{mM})$ were prepared by adding different amounts of a stock solution $(10 \mathrm{mM})$. A series of control solutions were also made by omitting the $\mathrm{CuCl} 2$. The flasks were shaken vigorously and left for $5 \mathrm{~min}$ to ensure reaction completion. Finally, the flasks were topped up with distilled water (total volume $10 \mathrm{~mL}$ ) and the absorbance at $478 \mathrm{~nm}$ measured. The results are shown in Figure SD3 and demonstrate that the absorbance of both the control solutions and the copper containing solutions increases linearly with PH concentration. However, the copper containing solutions reached a maximum absorbance at $0.25 \mathrm{mM} \mathrm{PH}$ after which the absorbance remained approximately constant. All further studies were conducted using $0.25 \mathrm{mM} \mathrm{PH}$. Then Jobs plot was constructed by plotting absorbance values against $[\mathrm{PH}] /[\mathrm{PH}]+[\mathrm{Cu} 2+]$ in order to know the binding ratio of $\mathrm{Cu} 2+$ ions and the probe from Figure SD4. It was found to be 1:1.pHAs previously discussed, the $\mathrm{UV}$-vis absorbance of $\mathrm{P}$ is known to be very sensitive toward $\mathrm{pH}$. Below $\mathrm{pH} 8.2 \mathrm{P}$ is colorless, becoming pink in $\mathrm{pH}$ range $8.2-12$, and returning colorless at $\mathrm{pH}$ 's greater than 12. These changes occur due to structural transformations that occur within the different $\mathrm{Ph}$ conditions. $\mathrm{PHs} \mathrm{pH}$ dependence is identical to the parent $\mathrm{P}$, but only having undergone hydrolysis by reacted with $\mathrm{Cu} 2+$ ions. If there has been no reaction, the molecule remains colorless across the entire $\mathrm{pH}$ range. Therefore, the effect of $\mathrm{Ph}$ on the absorbance of $\mathrm{PH}$ in solution was studied between $\mathrm{pH} 8-12$. Absolute $\mathrm{pH}$ optimization was done by keeping all other parameters constant. In a set of standard flasks $(10 \mathrm{~mL})$,Britton-Robinson buffer solution in the range $\mathrm{pH} 8-12(1 \mathrm{~mL})$ were prepared, to each $\mathrm{pH}$ solution $\mathrm{CuCl} 2(25 \mu \mathrm{M})$ and $\mathrm{PH}(0.25 \mathrm{mM}$, from $10 \mathrm{mM}$ stock solution, 1:10 acetonitrile : water)were added. The solutions were then left for $5 \mathrm{~min}$ to ensure reaction completion. Finally, the flasks were made up to 10 Ml with distilled water and the UV-vis absorbance recorded. From Figure SD5, it is clear that the absorbance of both the $\mathrm{PH}$ and $\mathrm{PH}$ reacting with $\mathrm{Cu} 2+$ ions increases as a function of $\mathrm{pH}$, with the maximum found to be at $\mathrm{pH} 12$. Therefore, further investigations of $\mathrm{PH}$ properties were tested in $\mathrm{pH} 12$ solutions. Although $\mathrm{pH} 12$ produced the largest difference in absorbance(sample absorbance-blank absorbance), it can be seen that this difference is similar for pH's 9-11, and there is still an appreciable difference when the $\mathrm{pH}$ was 7-8. Such results mean that the $\mathrm{PH}$ molecular probe is suitable for testing samples across all of these $\mathrm{pH}$ values, but the $\mathrm{pH}$ must be either known or fixed. Interestingly, there appears to be two regimes present, as the absorbance increases differently above $\mathrm{pH}$ 10on both plots. This observation was not probed further, as it is outside the scope of this research. However, these observations will be investigated in more detail in future studies of this and similar systems.

\section{Kinetic Studies}

The effect of time on the reaction between probe and $\mathrm{Cu} 2+$ ions has been investigated. In cuvettes $(3 \mathrm{~mL}), \mathrm{PH}(0.25 \mathrm{~mL}$ of $10 \mathrm{mM}$ stock solution (1:10 acetonitrile: water), $\mathrm{Cu} 2+$ ions 
$(25 \mu \mathrm{M})$, and Britton-Robinson buffer solutions, $\mathrm{pH} 12(1 \mathrm{~mL})$ was added and the absorbance was recorded as the function of time. This experiment was also repeated without the presence of $\mathrm{Cu} 2+$ ions. The results of these experiments are shown in Figure SD6. The absorbance values of both experiments change very little with time until circa. $200 \mathrm{~s}$, at which point there is a dramatic change in absorbance (at $478 \mathrm{~nm}$ ) for the $\mathrm{Cu} 2+$ ion treated sample. This then becomes constant approximately $60 \mathrm{~s}$ later. So $5 \mathrm{~min}$ is considered sufficient time to ensure the reaction has completed, allowing accurate absorbance values to be recorded.

\section{Interference Studies}

The selectivity of the probe $\mathrm{PH}$ with $\mathrm{Cu} 2+$ ions, under the determined optimum conditions was studied. The other metal ions tested were $\mathrm{Na}+\mathrm{Mg} 2+, \mathrm{Al} 3+, \mathrm{K}+, \mathrm{Ca} 2+, \mathrm{Hg} 2+, \mathrm{Pb} 2+$, $\mathrm{Fe} 2+, \mathrm{Fe} 3+, \mathrm{Ni2}+, \mathrm{Zn} 2+$, and $\mathrm{Cd} 2+$ and, with the exception of $\mathrm{Hg} 2+$ no significant changes in absorbance were observed (Figure 3and Figure SD7 in Supplementary data). No significant absorbance changes occurred even when the metal ions were present at significantly high concentrations (tested up to $50 \mu \mathrm{M}$ ). The $\mathrm{Hg} 2+$ ions induced a noticeable, but relatively small absorbance change. The change is shown in Figure 2, and is minor compared to that induced by the $\mathrm{Cu} 2+$ ions. The binding ofHg2+ ions can possibly be explained by the copper triangle[25]. Anions commonly present in water like $\mathrm{Cl}-$, $\mathrm{NO} 3-, \mathrm{NO} 2-, \mathrm{SO} 42-, \mathrm{F}-$, $\mathrm{CH} 3 \mathrm{COO}-$, and $\mathrm{PO} 43^{-}$were also studied and did not induce any observable color or measureable absorbance changes within the $\mathrm{PH}$ molecule probe, in any conditions tested (various concentrations, $\mathrm{pH} 1-12$ ). The absorbance changes of $1.0 \mu \mathrm{M} \mathrm{Cu} 2+$ ions in the presence of other cations and anions are given in Figure SD7 in supplementary data.

\section{Analytical Merits of the Sensor}

The proposed sensor system exhibited a good analytical performance for $\mathrm{Cu} 2+$ ions colorimetric sensing with a wide linear range $(0-22 \mu \mathrm{M})$, low limit of detection $(0.279 \mu \mathrm{M})$, good selectivity, and recovery studies. The performance of the sensor was similar or sometimes better compared to the some of the reported colorimetric sensor systems for $\mathrm{Cu} 2+$ ions(Table S1 Supplementary material [26-29]).

\section{Application Studies}

The $\mathrm{PH}$ molecular probe has been used to determine the presence of $\mathrm{Cu} 2+$ ions in environmental water samples, using the optimized conditions determined from the previous tests. The water samples were collected from the lakes that are in the vicinity of industrial manufacturing planets, and were found to contain about 10-15 ppm divalent copper. These results were compared to the analysis of tap water, which was found not to contain any detectable divalent copper. Further testing of the sensors was conducted by performing recovery studies. These involved spiking the real samples with known concentrations ofCu2+ ions, and comparing the known concentration values with the measured ones. The recovery was found to be greater than $99 \%$ in all cases. The PH molecular probe method was also validated by analyzing all the samples with AAS and fluorimetric methods in tandem [22]. The results of both these $\mathrm{Cu} 2+$ ion concentration determination-testing methods are presented in Table 1 . The obtained results were statistically compared with the Student-t test and variance ratio F-test with fluorimetric and AAS method results [22]. The Student t-test values did not exceed theoretical value sat $95 \%$ confidence level suggests that there is no significant difference between the results of proposed and fluorimetric/AAS methods [22]. Then variance 
ration $\mathrm{F}$ values $(\mathrm{P}=0.05)$ indicate there is no difference between the precessions of the two methods.

\section{Naked Eye Detection of Cu2+ Ions Using Polymer Membrane Test Strip}

The use of organic-inorganic hybrid materials for ion reorganization and sensing is a new area of research, attracting considerable amount of interest. Receptor-immobilized inorganic materials, such as $\mathrm{SiO} 2, \mathrm{Al} 2 \mathrm{O} 3$, and $\mathrm{TiO} 2$, have important advantages as solid chemosensors in heterogeneous solid-liquid phases [30]. Some of the organic-inorganic hybrid materials have been reported as metal ion sensors [31]. In this regard we attempted to design a polymer membrane strip for divalent copper ion detection. The membrane fabrication procedure is given in the procedure section. The polymer membrane fabricated, showed good selectivity toward the $\mathrm{Cu} 2+$ ions. When the membrane was dipped in solutions containing divalent copper ions the solution turned pink. When the membrane was dipped in probe solution as well as in solutions of other metal ions along with the probe, the solution is almost colorless (Figure SD9, Supplementary material). The color of the solutions in presence of membrane strip increases with increase in $\mathrm{Cu} 2+$ ions (Figure SD10, Supplementary material). The membrane strip has been successfully utilized forCu2+ion determination in various environmental samples(Figure SD11, Supplementary material). The fabricated membrane can be used for divalent copper monitoring in drinking water, because the threshold limit values of the copper in drinking water is $1.3 \mathrm{ppm}$. Nevertheless, the prepared test membrane can detect divalent copper ions less than these concentrations(below $1 \mathrm{ppm}$ ) [14]. The membrane strips operate by leaching out the $\mathrm{PH}$ at a steady rate when in an aqueous solution (due to the hydrophilic nature of the molecule), which subsequently complexes with any free $\mathrm{Cu} 2+$ ions. A strip is placed into a solution comprising of the sample $(4 \mathrm{~mL})$ and Robinson's buffer $\mathrm{pH} 12(1 \mathrm{~mL})$ and left for 10 min, with gentle mixing. After this time the absorbance can be measured and the concentration of $\mathrm{Cu} 2+$ in the sample determined. The strip scan then be rinsed and dried ready to be used again. Each strip can be used up to four times, after which the concentration of PH leaching from the strip is depleted and the response decreases from the correct value. The average UVVis absorbance $(478 \mathrm{~nm})$ of four separate $\mathrm{Cu} 2+$ ion solutions $(10 \mu \mathrm{M})$, measured consecutively was $0.5232\left(\_0.0005\right)$ a.u.

\section{CONCLUSIONS}

In this manuscript, we report design and synthesis of $\mathrm{PH}$ as a simple, sensitive, and selective colorimetric probe for divalent copper ions. The probe along with polysulfone was fabricated into a solid-state polymer membrane that could be used(with a known volume of water) as a solid-state sensing strip for the detection of divalent copper ions at micro-molar concentrations. The membrane strip can be readily utilized for monitoring the divalent copper ions in drinking water, because the strip can give color response with copper concentration less than the threshold limit value (1.3 ppm) set by the Environmental protection agency. Furthermore, the method was validated by comparing the obtained results with the AASmethod.

\section{ACKNOWLEDGMENTS}

The authors acknowledge Indian Institute of Science for providing the NMR and ESI-MS facilities and Jain University for financial support. S.M tanks to KMUTT for the 55th Anniversary Commemorative Fund. 


\section{References}

1. Hilal, N., Kochkodan, V., Abdulgader, H.A., Mandale, S., \& Jlil, S.A.A. (2015). A combined ion Exchange-nanofiltration process for water desalination: III. Pilot scale studies, Desalination, 363, 58-63.

2. Jyothi, M.S., Nayak, V., Padaki, M., Balakrishna, R.G., \& Ismail, A.F. (2014). The Effect of UV irradiation on $\mathrm{PSf} / \mathrm{TiO}_{2}$ mixed matrix for chromium rejection, Desalination, 354, 189-199.

3. Tao, Q., Zhou, S., Luo, J., \& Yuan, J. (2015). Nutrient removal and electricity production from wastewater using microbial fuel cell technique, Desalination, 365, 92-98.

4. Li, Z., Zhang, L., Amirkhiz, B.S., Tan, X., Xu, Z., Wang, H., Olsen, B.C., Holt, C.M.B., \& Mitlin, D. (2012). Carbonized Chicken Eggshell Membranes with 3D Architectures as High Performance Electrode Materials for Supercapacitors, Advanced Energy Materials, 2, 431-437.

5. Duncan, T.V. (2011). Applications of nanotechnology in food packaging and food safety: Barrier materials,

antimicrobials and sensors, Journal of Colloid and Interface

Science, 363, 1-24.

6. Qiu, Y., \& Park, K. (2012). Environment-sensitive hydrogels for drug deliver, Advanced Drug Delivery Reviews, 64, 49-60.

7. He, L., Dumee, L.F., Feng, C., Velleman, L., Reis, R., She, F., Gao, W., \& Kong, L. (2015). Promoted water transport across graphene oxide-poly(amide) thin film composite membranes and their antibacterial activity, Desalination, 365, 126-135.

8. Guo, H., Chen, M., Liu, Q., Wang, Z., Cui, S., \& Zhang, G. (2015). LbL assembly of sulfonated cyclohexanone-formaldehyde condensation polymer and poly(ethyleneimine)

towards rejection of both cationic ions and dyes, Desalination, 365, 108-116.

9. Ang, W.L., Mohammad, A.W., Hilal, N., \& Leo, C.P. (2015). A review on the applicability of integrated/hybrid membrane processes in water treatment and desalination plants, Desalination, 363, 2-18.

10. Wang, Q.R., Kim, D., Dionysiou, D.D., Sorial, G.A., \& Timberlake, D. (2004). Sources and remediation for mercury contamination in aquatic systems-a literature review, Environmental Pollution, 131, 323-336.

11. Krupanidhi, S., Sreekumar, A., \& Sanjeevi, C.B. (2008). Copper \& biological health, The Indian Journal of Medical Research, 128, 448-461.

12. Que, E.L., Domaille, D.W., \& Chang, C.J. (2008). Metals in neurobiology: Probing their chemistry and biology with molecular imaging, Chemical Reviews, 108, 1517-1549.

13. Harris, E.D. (2001). Copper and Iron: A landmark connection of two essential metals, Journal of Trace Elements in Experimental Medicine, 14, 207-210.

14. Hahn, S.H., Tanner, M.S., Danke, D.M., \& Gahl, W.A. (1995). Normal metallothione in synthesis in fibroblasts obtained from children with indian childhood cirrhosis or copper-associated childhood cirrhosis, Biochemical and Molecular Medicine, 54, 142-145.

15. Zhao, Y., Zhang, X.B., Han, Z.X., Qiao, L., Li, C.Y., Jian, L. X., Shen, G.L., \& Yu, R.Q. (2009). Highly sensitive and selective colorimetric and off-on fluorescent chemosensor for $\mathrm{Cu}_{2+}$ in aqueous solution and living cells, Analytical Chemistry, 81, 7022-7030.

16. Lan, G.Y., Huang, C.C., \& Chang, H.T. (2010). Silver nanoclusters as fluorescent probes for selective and sensitive detection of copper ions, Chemical Communications, 46, 1257-1259. 17. Leermakers, M., Baeyens, W., Quevauviller, P., \& Horvat, M. 
(2005). Mercury in environmental samples: Speciation, artifacts and validation, Trends in Analytical Chemistry, 24, 383-393. 18. Valeur, B. (2002). Molecular Fluorescence: Principles and Applications, Germany: Wiley-VCH Weinheim.

19. Kaur, P., Sareena, D., \& Singh, K. (2011). Selective colorimetric sensing of $\mathrm{Cu} 2+$ using triazolyl monoazo derivative,

Talanta, 83, 1695-1700.

Quang, D.T., \& Kim, J.S. (2010). Fluoro and chromogenic chemodosimeters for heavy metal ion detection in solution and biospecimens, Chemical Reviews, 110, 6280-6301. 21. Yin, S., Leen, V., Snick, S.V., Boensa, N., \& Dehaena, W. (2010). A highly sensitive, selective, colorimetric and nearinfrared fluorescent turn-on chemosensor for $\mathrm{Cu}_{2+}$ based on BODIPY, Chemical Communications, 46, 6329-6331.

22. Kempahanumakkagaari, S.K., Thippeswamy, R., \& Malingappa, P. (2014). A new rhodamine B based fluorometric chemodosimeter for $\mathrm{Cu}_{2}+$ ion in aqueous and cellular media, Journal of Luminescence, 146, 11-17.

23. Xiaogiang, C., Tuhin, P., Fang, W., Jong, S., \& Juyoung, Y. (2012). Fluorescent chemosensors based on spiroringopening of xanthenes and related derivatives, Chemical

Reviews, 112, 1910-1956.

24. Pandurangappa, M., \& Sureshkumar, K. (2011). Micellar mediated trace level mercury quantification through the rhodamine $B$ hydrazide spirolactam ring opening process, Analytical Methods, 3, 715-723.

25. Copper, T.H., Mayer, M.J., Leung, K.H., Ochrymowycz, L. A., \& Rorabacher, D.B. (1992). Kinetic and thermodynamic measurements on branched amino polythiaether ligands: a family of complexing agents analogous to EDTA and NTA exhibiting enhanced selectivity for copper(II), Inorganic Chemistry, 31, 3796-3804.

26. Domínguez, D.P., Rodriguez, M., Ortiz, G.R., Maldonado, J.L., Moreno, D.L., Gutierrez, M.O., \& Barba, V. (2016). A Schiff base derivative used as sensor of copper through colorimetric and surface plasmon resonance techniques, Sensors and Actuators B: Chemical, 225, 221-227. 27. Ozdemir, M. (2006). A rhodamine-based colorimetric and fluorescent probe for dual sensing of $\mathrm{Cu}_{2+}$ and $\mathrm{Hg}_{2+}$ ions, Journal of Photochemistry and Photobiology A: Chemistry, 318, 7-13.

28. Li, M., Sun, Y., Dong, L., Feng, Q.C., Xu, H., Zang, S. Q., \& Mak, T.C.W. (2016). Colorimetric recognition of $\mathrm{Cu}_{2+}$ and fluorescent detection of $\mathrm{Hg}_{2+}$ in aqueous media by a dual chemosensor derived from rhodamine $B$ dye with a NS2 receptor, Sensors and Actuators B: Chemical, 226, 332-341.

29. Gao, Y., Zhang, C., Peng, S., \& Chen, H. (2017). A fluorescent and colorimetric probe enables simultaneous differential detection of $\mathrm{Hg}_{2+}$ and $\mathrm{Cu}_{2+}$ by two different mechanisms, Sensors and Actuators B: Chemical, 238, 455-461.

30. Wight, A.P., \& Davis, M.E. (2002). Design and preparation of organic-inorganic hybrid catalysts, Chemical Reviews, 102, 3589-3614.

31. Lee, M.H., Lee, S.J., Jung, J.H., Lim, H., \& Kim, J.S. (2007). Luminophore-immobilized mesoporous silica for selective $\mathrm{Hg}_{2+}$ sensing, Tetrahedron, 63, 12087-12092. 


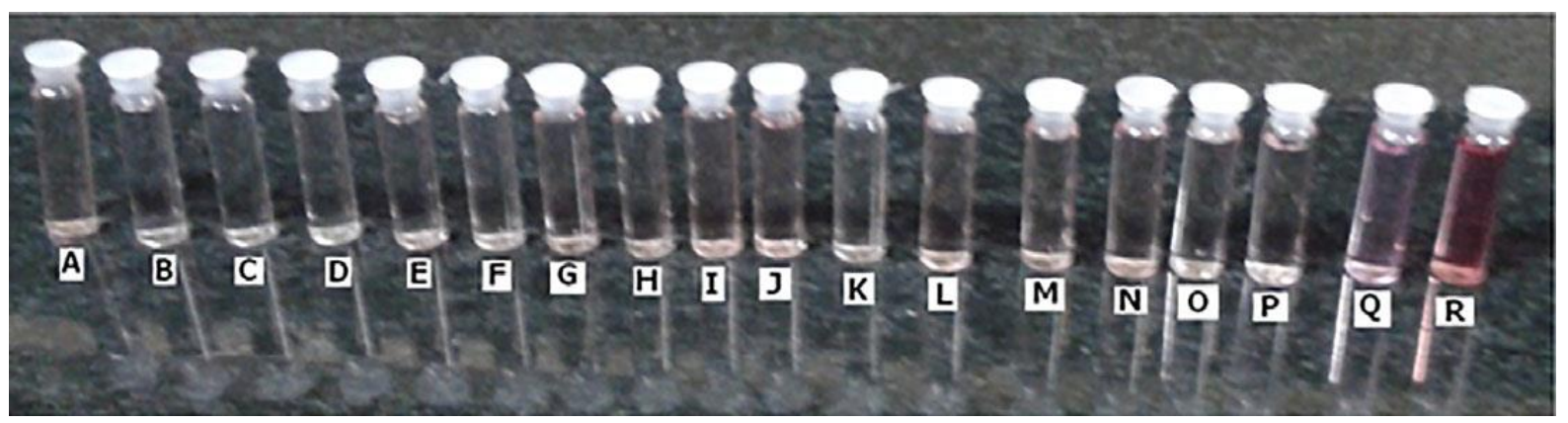

Figure 3. Photographs of color changes of the $\mathrm{PH}(0.25 \mu \mathrm{M}$ in $1: 10$ acetonitrile water in $\mathrm{pH} 12$ Britton-Robinson buffer solution)

with different metal ions ( $3 \mathrm{~mL}$ of corresponding metal nitrite solutions of $100 \mathrm{ppm}$ dissolved in water) (a) Probe alone, (b) $\mathrm{Pb}_{2+}$

ions, (c) $\mathrm{Cd}_{2+}$ ions, (d) $\mathrm{Ca}_{2+}$ ions, (e) $\mathrm{Ni}_{2+}$ ions, (f ) $\mathrm{Mg}_{2+}$ ions, (g) $\mathrm{Zn}_{2+}$ ions, (h) $\mathrm{K}_{+}$ions, (i) $\mathrm{Fe}_{2+}$ ions, (j) Fe $3+$ ions, (k) $\mathrm{Al}_{3+\text { ions, }}$

(l) Cr6+ions, (m) Co2+ions, (n) Ag+ions, (o) Cr3+ions, (p) Mn2+ions, (q) Hg2+ ions, and (r) Cu2+ ions. [Color figure can be viewed

at wileyonlinelibrary.com]

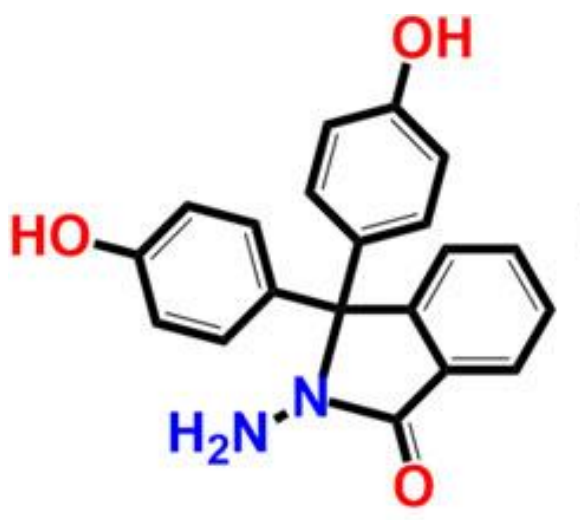

$[\mathrm{M}+\mathrm{H}]^{+}=333.2$

Phenolphthalein hydrazide- Colorless

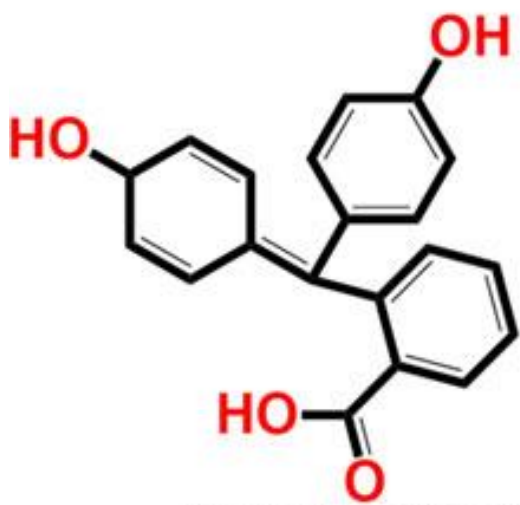

$[\mathrm{M}+\mathrm{H}]^{+}=321.3$

\section{Phenolphthalein- Pink}

Scheme 3. Proposed reaction mechanism of phenolphthalein hydrazide with $\mathrm{Cu}_{2+}$ ions. [Color figure can be viewed at wileyonlinelibrary.com]
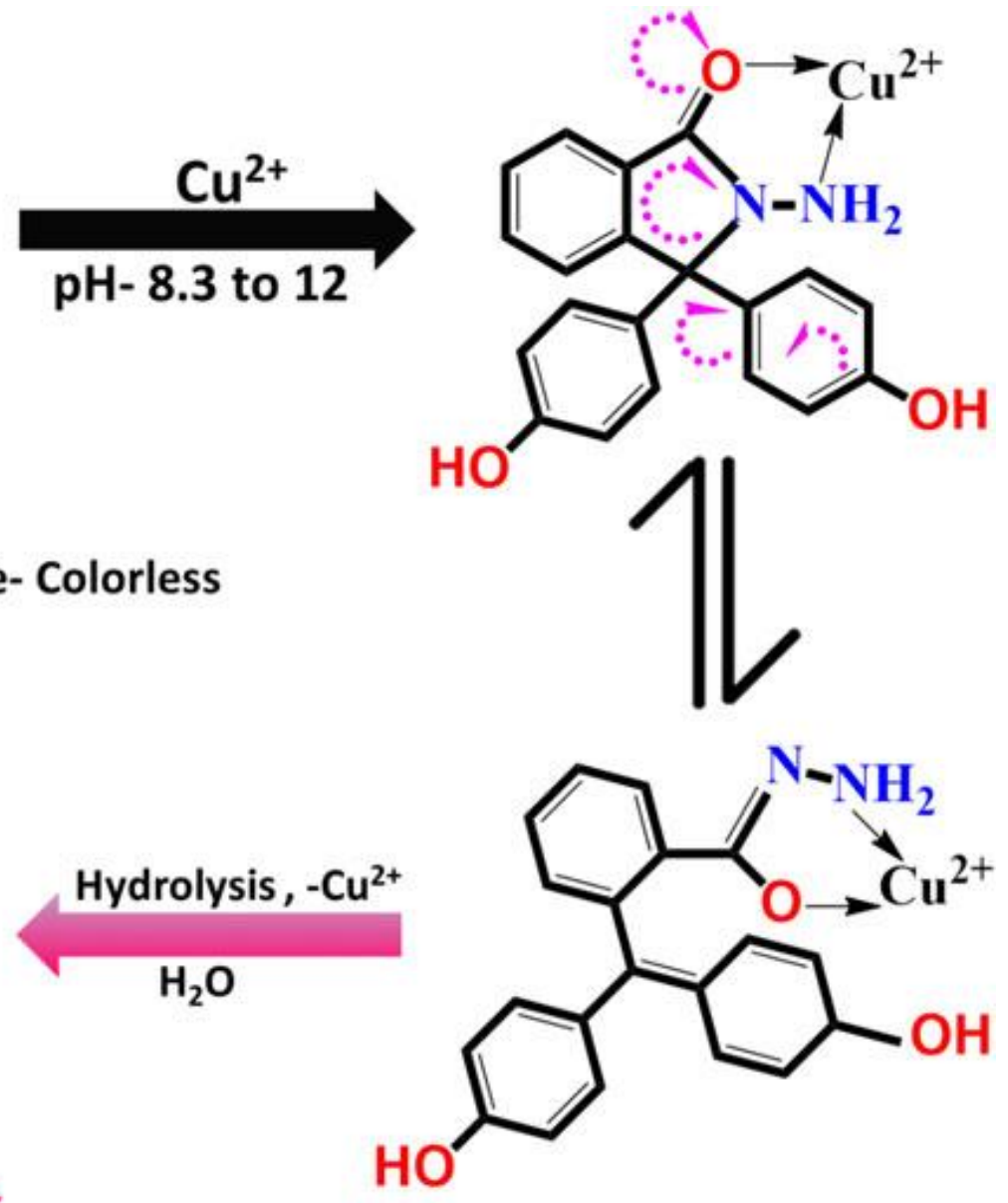


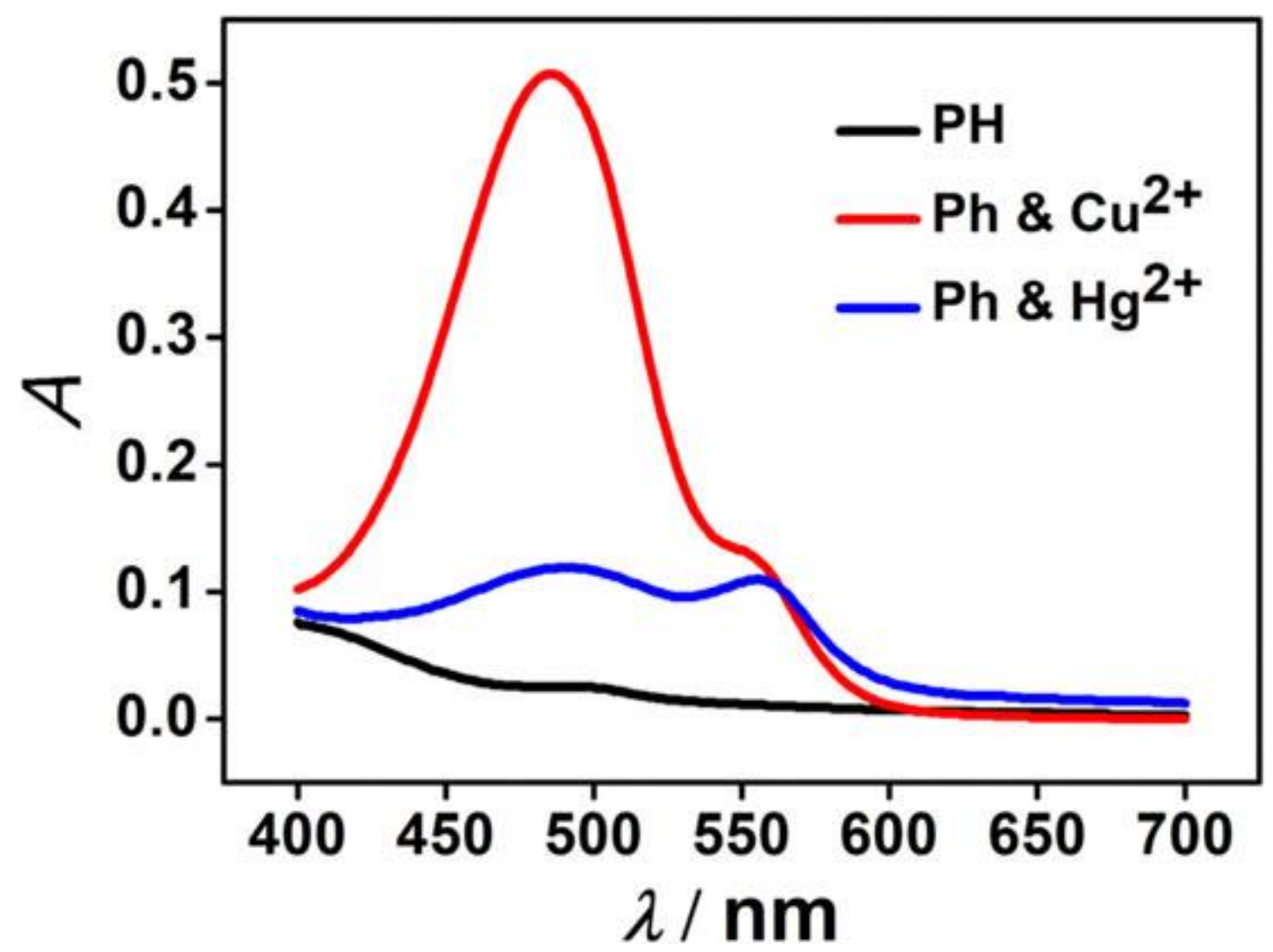

Figure 2. Absorption spectra of $\mathrm{PH}(50 \mu \mathrm{M}, 1: 10$ acetonitrile water in $\mathrm{pH} 12$ Britton-Robinson buffer solution) with (black line) probe alone (blue line) $50 \mu \mathrm{M} \mathrm{Hg}_{2+}$ ions and (redline) $50 \mu \mathrm{M}$ Cu2+ ions. [Color figure can be viewed at wileyonlinelibrary.com]
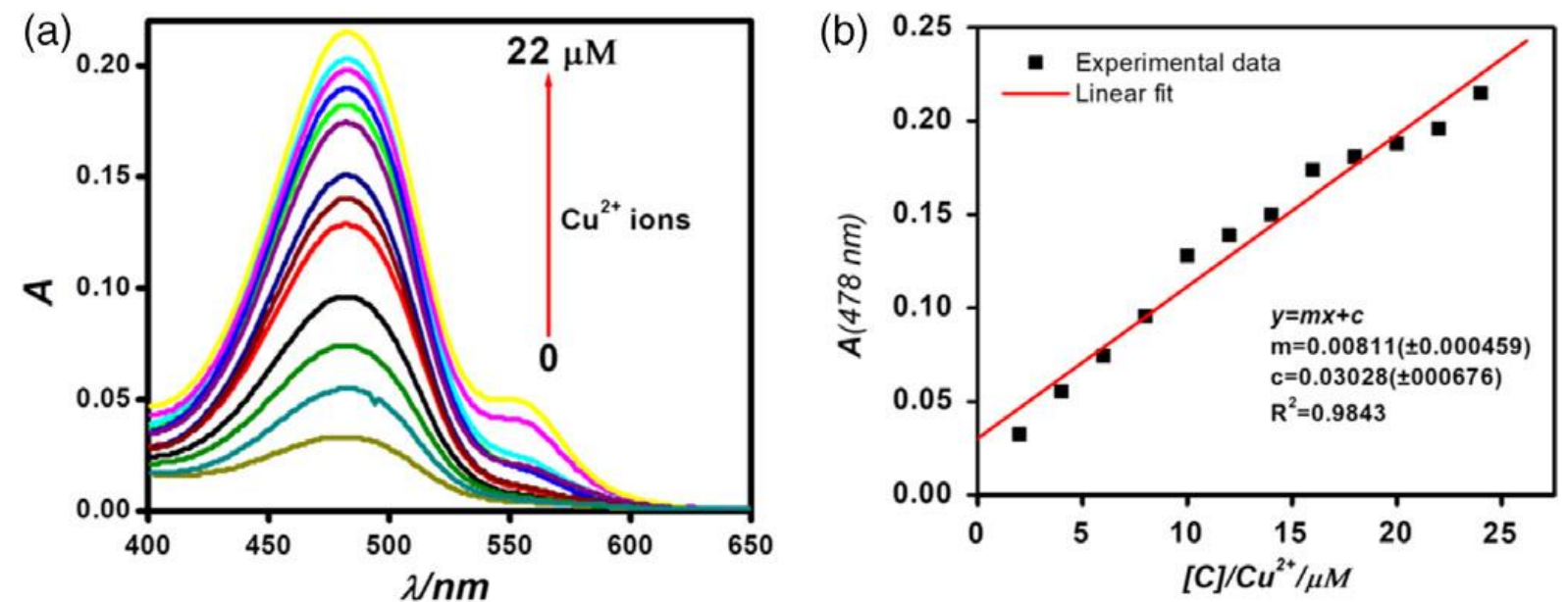

Figure 1. (a) Absorption spectra of $\mathrm{PH}$ in acetonitrile: water (1:10) solution $\mathrm{pH} 12$ Britton-Robinson buffer solution with

increasing concentrations of $\mathrm{Cu}_{2+}$ ions $(0-22 \mu \mathrm{M})$. (b) Corresponding calibration plot. [Color figure can be viewed at

wileyonlinelibrary.com] 

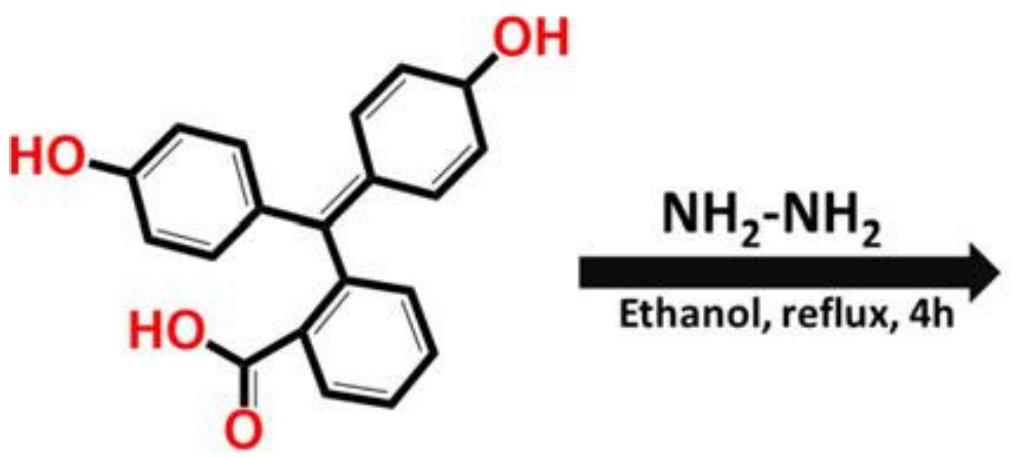

Scheme 1. Synthetic route to $\mathrm{PH}$. [Color figure can be viewed at wileyonlinelibrary.com]

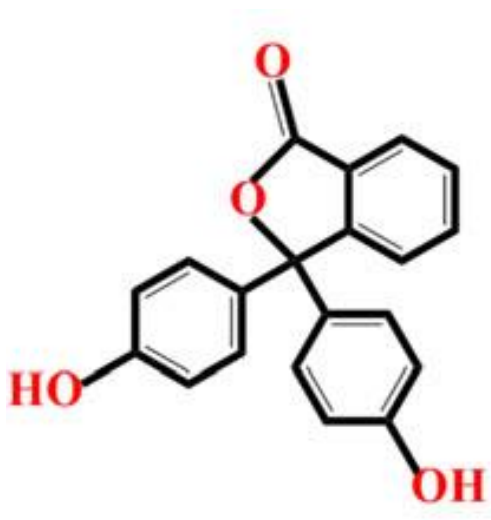

pH 1 to 8.2

Colorless

Ring closed form

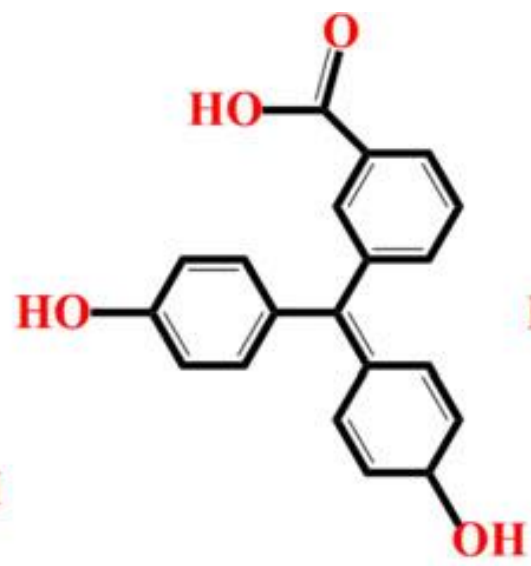

$\mathrm{pH} 8.2$ to 12

Pink

Ring opened form

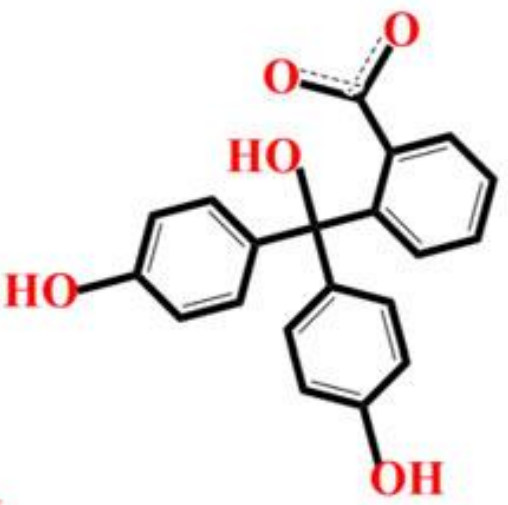

$\mathrm{pH} \geq 12$

Color less

Ring opened carbinol form

Scheme 2. The structures of phenolphthalein and their associated color in different $\mathrm{pH}$ conditions. [Color figure can be viewed at wileyonlinelibrary.com] 